\title{
COMPOSICIÓN FLORÍSTICA Y VEGETACIÓN DE UNA MICROCUENCA ANDINA: EL PACHACHACA (HUANCAVELICA)
}

\section{FLORISTIC COMPOSITION AND VEGETATION OF AN ANDEAN MICROWATERSHED: EL PACHACHACA (HUANCAVELICA)}

\author{
Fabiola Parra Rondinel ${ }^{1}$, Juan Torres Guevara ${ }^{1}$ y Aldo Ceroni Stuva ${ }^{1}$
}

\section{Resumen}

Se estudió la composición florística y la vegetación de la microcuenca del Pachachaca, ubicada al noroeste del Departamento de Huancavelica, y realizado entre el año 2001 y 2003. Se determinaron 180 especies pertenecientes a 57 familias. En cuanto al tipo de formas de vida, dominan las herbáceas y existen muy pocas arbóreas, sobre todo nativas. Se identificaron 8 formaciones vegetales naturales representativas, entre oconales y pajonales en la parte alta y matorrales en casi toda la microcuenca. La composición florística de estas formaciones vegetales demuestra una gran riqueza específica, siendo las familias mejor representadas, Asteraceae en las partes medias y bajas, Poaceae en las partes altas y Fabaceae en las partes medias y bajas de la microcuenca.

Palabras clave: composición florística, formaciones vegetales, oconales, pajonales, matorrales.

\begin{abstract}
Vegetation and floristic composition of the Pachachaca basin, located in north western Huancavelica were studied from 2001 to 2003. There were registered 180 species belonging to 57 families. Herbaceous life forms dominated over other life forms, and only a few native tree species were registered. Eight representative natural plant formations were identified between grasslands and humid, compact slow-growing vegetation named oconales in the high areas, and shrub vegetation almost all over the basin. Floristic composition shows a large richness in species. Asteraceae were most representative in the middle and lowland areas, Poaceae in the high lands, and Fabaceae in the middle and lowland areas of the watershed.
\end{abstract}

Key words: floristic composition, plant formations, oconales, grass lands, shrub vegetation.

\section{Introducción}

En la conservación de los ecosistemas montañosos altoandinos la cobertura vegetal constituye un elemento central. La microcuenca del río Pachachaca es un ecosistema de montaña y uno de los tres ríos afluentes del río Cachi que a su vez forma parte de la más importante cuenca de la Sierra Central Sur del Perú: el Mantaro. Este ecosistema presenta una variedad de formaciones vegetales representativas de la Sierra Central Sur del Perú y en especial presenta importantes áreas de humedales en sus partes más altas, denominadas oconales o bofedales, formaciones vegetales de gran importancia para el ciclo hidrológico de la misma, algo muy frecuente en los territorios altoandinos de nuestro país. También presenta grandes extensiones de pastos naturales, uno de los mayores recursos con los que cuenta el departamento de Huancavelica, con más de 820000 ha de su superficie. Las contribuciones al conocimiento sobre la composición florística y su distribución en Huancavelica tiene como principal representante a Tovar (1957, 1990, 1993), siendo su mayor aporte en lo referido a la familia Poaceae. Es tal vez el único que ha realizado investigaciones florísticas en algunos puntos de la microcuenca referida en el presente estudio. Ochoa (1999) ha llevado a cabo numerosas colectas de papas silvestres en Huancavelica. Trabajos adicionales como el de la ONERN (1984), antigua oficina del Instituto Nacional de Recursos Naturales (INRENA), también han contribuido al conocimiento del tema. A su vez es renombrable el esfuerzo llevado a cabo por Desco, al elaborar con la colaboración de Barreda \& Rubina (2000) el Atlas de Huancavelica, el cual constituye otro de los aportes al conocimiento de la diversidad de la Región. Como trabajo de base para todos los mencionados, es el realizado por Weberbauer (1945), quien llevó a cabo colecciones en el Valle del Mantaro, muy cerca al área de estudio. El conocimiento que se tiene sobre la Flora del Departamento de Huancavelica, es aún limitado, por lo que es necesario determinar las especies vegetales que componen a este gran ecosistema montañoso, y con ello, identificar zonas de cobertura y estructura más compleja, que nos puedan ofrecer información sobre la composición de estos, y su posible potencial económico, aun no reconocidos, con la finalidad de conservarlos usándolos adecuadamente. El presente trabajo es una contribución al conocimiento de la composición florística y de las formaciones vegetales representativas de la microcuenca del Pachachaca, 
como una muestra de lo que es la vegetación de la Sierra Central Sur del Perú.

\section{Materiales y métodos \\ Ubicación}

La microcuenca del río Pachachaca está ubicada en los Distritos de Laria y de Nuevo Occoro, Provincia de Huancavelica, al noroeste del Departamento de Huacavelica, entre los $12^{\circ} 32^{\prime}$ y $12^{\circ} 37^{\prime}$ LS y entre los $75^{\circ} 6^{\prime}$ y $75^{\circ} 1^{\prime}$ LO. Hidrográficamente, se ubica como afluente de primer grado de la cuenca del río Cachi junto con los ríos Alauma y el Occoro que a su vez forman parte de la gran cuenca del río Mantaro (Figura 1).
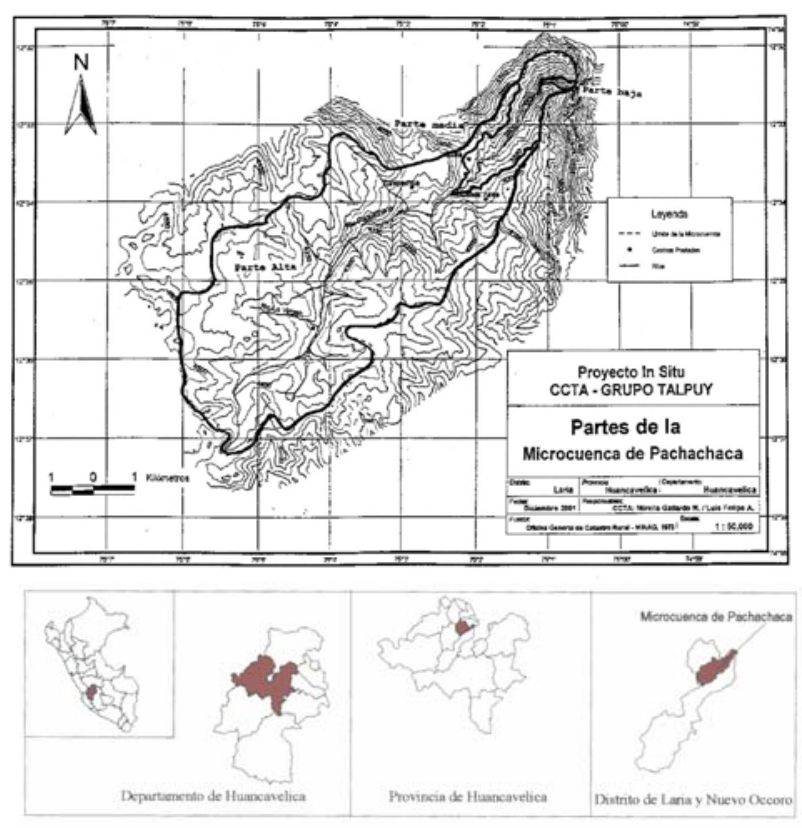

Figura 1. Microcuenca del río Pachachaca.

Huancavelica.

\section{Caracterización de la zona de estudio Área}

La microcuenca tiene un área aproximada de 3606.7 ha, extensión que la tipifica como microcuenca. El área de estudio puede ser dividida en tres zonas: baja, entre 3400 y 3550 msnm; media, entre 3550 y 3900 msnm; y alta, entre 3900 y 4460 msnm.

Clima

El clima está determinado por la presencia o ausencia de lluvias. El inicio de las lluvias o época de "invierno", se da en Octubre, finalizando cerca al mes de Mayo, siendo el resto del año meses secos o "verano". La temperatura oscila desde menos de $0^{\circ} \mathrm{C}$ hasta $30^{\circ} \mathrm{C}$, de la noche al día en un mes verano. El clima es bastante seco, disminuyendo la humedad a medida que se sube a lo largo de la microcuenca.

\section{Suelos}

En toda la microcuenca el suelo es primordialmente franco, con un $\mathrm{pH}$ que va desde 5.8 a 7.4 aproximada-mente, una conductividad eléctrica no mayor a 0.20 mmhos, indicando un bajo nivel de sales, debido al lavado que sufre el suelo en cada estación húmeda. La erosión es debido a la actividad geo-dinámica como en las pendientes pronunciadas donde masas de tierra son acarreadas en época de lluvias. La presión del uso del suelo por la población ha ido en aumento junto con el incremento en el número de habitantes, una explotación excesiva de las tierras que quedaban en descanso por más de 7 años y que hoy en día no descansan más de 3 años. Cultivos en áreas de excesiva pendiente no permiten la regeneración de la vegetación nativa, que protege de la erosión (Talpuy-UNALM, 2001).

\section{Fisiografía}

La microcuenca presenta pendientes pronunciadas de laderas donde llegan a tener hasta menos de $15^{\circ}$. Las formaciones rocosas de las laderas también contribuyen a esta característica. La parte alta de la microcuenca forma una planicie ondulada cubierta por bofedales. La parte media y baja está formada por cerros de laderas abruptas, que forman cañones aproximadamente entre los 3900 y $4100 \mathrm{msnm}$ y entre los 3500 y $3700 \mathrm{msnm}$.

\section{Ecología}

De acuerdo al Mapa Ecológico del Perú (ONERN, 1976) elaborado en base a las zonas de vida de Tosi (1960), la microcuenca del Pachachaca se encuentra comprendida entre 3 zonas de vida: tundra pluvialAlpino Tropical (tp-AT),páramo muy húmedoSubalpino Tropical (pmh-SaT), y el bosque húmedoMontano Tropical (bh-MT). Pero cabe recalcar que debido a la diversidad de ecosistemas que se pueden encontrar en cada zona de vida, existen tipos de clasificación más precisas en cuanto a vegetación, para el caso del Perú, como la usada por Martínez et al. (1989), o por Weberbauer (1945).

\section{Metodología}

Se evaluó la composición florística realizando colectas durante la época seca y época húmeda, debido a que en estas dos estaciones aparecen especies diferentes. Se colectaron las muestras procurando seguir transectos de 100 metros. Las muestras fueron determinadas en el Herbario USM de la Universidad Nacional Mayor de San Marcos, y en el Herbario MOL de la Universidad Nacional Agraria La Molina, con el apoyo de taxónomos reconocidos y la orientación de catálogos como el de los autores Brako \& Zarucchi (1993). Se identificaron las formaciones vegetales realizando evaluaciones a lo largo de la microcuenca siguiendo el tipo de muestreo utilizado por Torres (1987) y Vila (1997), y nombrando cada formación según la dominancia de algún tipo de forma de vida, por la textura, e identificando a su vez las 
especies dominantes, según la metodología usada por Parra (2003). El matorral es definido como la formación dominada por la presencia de arbustos de diferentes texturas de hojas; el pajonal por especies de los géneros Festuca, Stipa, Calamagrostis y Poa, que son gramíneas de hojas duras y punzantes (Tovar, 1993); el pastizal o césped de Puna constituido por hierbas específicamente gramíneas de porte reducido y juncáceas; y los oconales, como formaciones dominadas por especies almohadilladas, arrosetadas o de porte reducido, específicamente por juncáceas y gramíneas. Para definir la altura de los matorrales medios se tomaron rangos de 1 a $2.5 \mathrm{~m}$; para los bajos de 0.25 a $1 \mathrm{~m}$; y para los enanos, alturas menores a $0.25 \mathrm{~m}$. Para las texturas de las hojas se definió inerme al haber más del $70 \%$ de individuos con estructuras desprovistas de espinas o estructuras punzantes; subinerme con menos del $50 \%$ de individuos con estructuras punzantes; sub-espinoso entre $50 \%$ y $70 \%$ de individuos con estructuras punzantes, y espinoso con más del $70 \%$ de los individuos con estructuras punzantes. No se tomaron en cuenta formaciones arbóreas debido a no hallarse en condiciones naturales.

\section{Resultados y discusión Composición Florística}

Los resultados de la composición florística nos dan una idea de la diversidad y riqueza florística existente en la microcuenca del Pachachaca. Se hallaron un total de 179 especies pertenecientes a 121 géneros y 57 familias botánicas (Tabla 1). Altitudinalmente, es la zona baja la que posee una mayor diversidad, determinándose alrededor del $49 \%$, $57 \%$ y $67 \%$ del total de especies, géneros y familias, respectivamente, del total existente en la microcuenca. Las familias mejor representadas fueron: Asteraceae, Poaceae y Fabaceae, con 30, 23 y 10 especies, respectivamente (Figura 2). La familia Asteraceae incluye especies aromáticas (condimenticias) como Tagetes eliptica "huacatay", diversas especies de Baccharis, como B. latifolia o B. salicifolia, conocidas comúnmente como "chilca", o B. genistelloides "tres esquinas" y a Chuquiraga spinosa "huamanpinta". La familia Poaceae constituyen en su gran mayoría a los pastizales y pajonales, tales como Stipa ichu y Festuca weberbaueri, así como Calamagrostis vicunarum, Festuca breviaristata y Aciachne pulvinata. La familia, Fabaceae, cuenta con especies del género Senna, usadas como cerco vivo; o Astragalus garbancillo "garbancillo", de amplia distribución en la cuenca. Las familias representadas por una sola especie o mono específicas, fueron en total 29 , y aquellas representadas por 2 especies fueron 12 . Un aspecto importante es la presencia de ciertas especies reconocidas científicamente y por las poblaciones locales, como parientes silvestres de cultivos nativos de la zona. Tal es el caso de especies como Solanum bukasovii, Solanum acaule y Solanum multiinterruptum, parientes silvestres de las papas cultivadas de las especies tuberosas del género Solanum; Oxalis aff. peduncularis, Oxalis aff. nubigena, parientes de la oca cultivada Oxalis tuberosa y Tropaeolum tuberosum var. silvestre, pariente de la mashua Tropaeolum tuberosum.

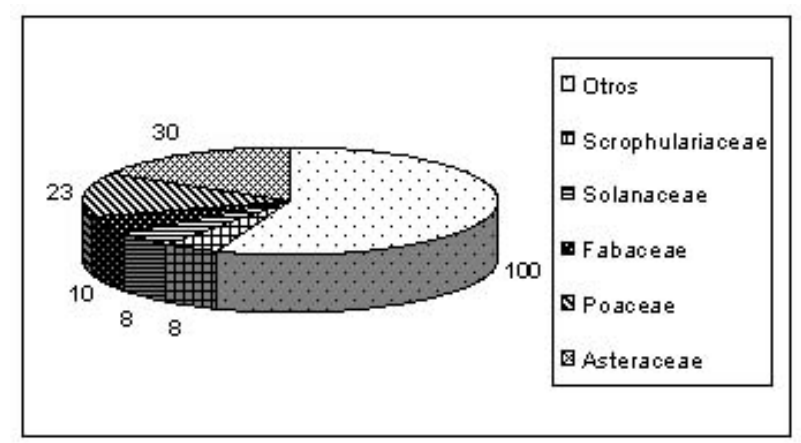

Figura 2.Número de especies de las familias mejor representadas en la microcuenca del río Pachachaca. Huancavelica.

\section{Formas de Vida}

La vegetación herbácea dominante en la microcuenca está representada por los pastos de la parte alta los cuales forman grandes extensiones de pajonales y pastizales, constituidas principalmente por especies de los géneros Calamagrostis, Festuca y Stipa. Así mismo, las partes medias y bajas presentan una amplia riqueza específica de hierbas de diversas familias. La vegetación arbustiva también es abundante en la microcuenca, conformada mayormente por especies espinosas o no palatables, de acuerdo a su textura, que constituyen los matorrales, observándose que Duranta aff. armata, Colletia spinosissima, Dunalia aff. spinosa y especies del género Berberis, se encuentran en abudancia en las partes baja y media de la microcuenca. En las partes altas se pueden encontrar matorrales de textura lisa (inermes) en su mayoría. En cuanto a la vegetación arbórea, se han registrado y determinado en la parte media de la microcuenca la especie Buddleia incana y en las partes altas se registró la presencia de Buddleia coriacea "colle", como cercos vivos. En el caso de Polylepis racemosa, "queñoal", este se encuentra formando pequeños rodales a lo largo del cauce del río, siendo la mayor parte de individuos plantados en los bordes de chacra como cerco vivo. La mayor extensión de plantaciones forestales corresponde a la especie Eucalyptus globulus, la que fue introducida durante los proyectos de reforestación de los años 70 , y que ha ido reemplazando a las especies nativas antes mencionadas. Otros tipos de hábitos reconocidos fueron: epífitas y suculentas, tanto en las partes baja, media y alta de la microcuenca (Tabla 2). Las formas de vida reconocidas en el presente trabajo, pueden ser encontradas en las tres partes de la misma o en dos 
partes, lo que indica su amplia adaptación a diferentes condiciones microclimáticas. Por ejemplo, tenemos a Baccharis aff. caespitosa y Arcytophyllum thymifolium, que se les halla en las partes medias y altas, o Astragalus garbancillo que está distribuido en toda la microcuenca.

Tabla 1. Lista de especies colectadas en la microcuenca del Pachachaca. Huancavelica.

\begin{tabular}{|c|c|}
\hline FAMILIA/GÉNERO/ESPECIE & NOMBRE COMÚN \\
\hline \multicolumn{2}{|l|}{ AGAVACEAE } \\
\hline Agave americana $\mathrm{L}$. & agave \\
\hline \multicolumn{2}{|l|}{ AMARANTHACEAE } \\
\hline \multicolumn{2}{|l|}{ Althernanthera sp. } \\
\hline Gomphrena sp. & bottom-botom \\
\hline \multicolumn{2}{|l|}{ AMARYLLIDACEAE } \\
\hline Stenomesson sp. & yungay \\
\hline \multicolumn{2}{|l|}{ APIACEAE } \\
\hline Daucus montanus R. et P. & secopata \\
\hline \multicolumn{2}{|l|}{ ASPLENIACEAE } \\
\hline Asplenium peruvianum (C.presl) Hier. & culantro de pozo \\
\hline \multicolumn{2}{|l|}{ Asplenium haenkeamum(C.Presl) Hier. } \\
\hline \multicolumn{2}{|l|}{ Asplenium resiliens Kunze } \\
\hline \multicolumn{2}{|l|}{ ASTERACEAE } \\
\hline Achyrocline alata (Kunth.) D.C. & árnica \\
\hline Achyrocline spp. & manzanilla japonesa \\
\hline $\begin{array}{l}\text { Ageratina azangaroensis (Bip.ex Wedd) } \\
\text { K\&H. Rob. }\end{array}$ & huayrasacha \\
\hline Ambrosia arborescens Mill. & marco \\
\hline $\begin{array}{l}\text { Aqeratina aff sternbergiana (DC.) R.M. K. } \\
\text { \& H. Rob. }\end{array}$ & marmaquilla \\
\hline \multicolumn{2}{|l|}{ Aqeratina sp. } \\
\hline \multicolumn{2}{|l|}{ Aristeguieta discolor (D.C.) K\&R. } \\
\hline Baccharis aff.caespitosa (R. et P.) Pers. & Taya \\
\hline Baccharis aff.tricuneata (L.f.) Pers. & Taya \\
\hline Baccharis genistelloides (Lam.) Pers. & qensa sencca \\
\hline Baccharis latifolia (R. et P.) Pers. & chilca \\
\hline Baccharis aff.salicifolia (Ref.) Pers. & chilca \\
\hline Baccharis sp. & taya hembra \\
\hline Bidens triplinervia H.B.K. & chinasilcao \\
\hline \multicolumn{2}{|l|}{ Chuquiraga sp. } \\
\hline Chuquiraga spinosa Less. & huamanpinta \\
\hline Cosmos peucedanifolius Wedd. & para-para \\
\hline Gynoxys longifolia Wedd. & toccarhui \\
\hline Heliopsis aff.canescens Kunth. & masaccarcco macho \\
\hline Hypochoeris sp. & pañas \\
\hline Mutisia acuminata $\mathrm{R}$. et $\mathrm{P}$. & mancapaqui \\
\hline Perezia multiflora (H.K.B.) Less. & escorzonera \\
\hline Senecio collinus D.C. & maycha \\
\hline
\end{tabular}

\begin{tabular}{|c|c|}
\hline Senecio nutans Sch. Bip. & chachacoma \\
\hline Senecio rudbeackiaefolius. & yanamaycha \\
\hline Sonchus asper (L.)Hill. & iscaña \\
\hline Stevia macbridei Rob. & masaccarcco \\
\hline Tagetes elliptica Smith. & huacatay \\
\hline Vasquezia opposifolia. & castilla Marco \\
\hline \multicolumn{2}{|l|}{ Viguiera aff.procumbens (Pers.) S.F. Blake } \\
\hline \multicolumn{2}{|l|}{ BASELLACEAE } \\
\hline Ullucus sp. & K'ita ullucu \\
\hline \multicolumn{2}{|l|}{ BERBERIDACEAE } \\
\hline Berberis flexuosa R. et P. & ayrampo \\
\hline Berberis lutea $\mathrm{R}$. et $\mathrm{P}$. & checcche \\
\hline \multicolumn{2}{|l|}{ BRASSICACEAE } \\
\hline Brassica rapa ssp. campestris L. & yuyo \\
\hline \multicolumn{2}{|l|}{ Descurainia $\mathrm{sp.}$} \\
\hline Lepidium chichicara Desv. & chichicara \\
\hline Rorippa sp. & berro \\
\hline \multicolumn{2}{|l|}{ BROMELIACEAE } \\
\hline Puya sp. & uña de gato \\
\hline \multicolumn{2}{|l|}{ Tillandsia aff. paleacea. } \\
\hline \multicolumn{2}{|l|}{ Tillandsia capillaris $\mathrm{R}$. et $\mathrm{P}$. } \\
\hline Tillandsia sp. & tullma \\
\hline Tillandsia usneoides (L.) L. & ccaccahuayllo \\
\hline Tillandsia walteri Mez. & huicclla \\
\hline \multicolumn{2}{|l|}{ CACTACEAE } \\
\hline $\begin{array}{l}\text { Austrocylindropuntia floccosa (Salm-Dyck) } \\
\text { Ritt. }\end{array}$ & ccalahuaracco \\
\hline $\begin{array}{l}\text { Austrocylindropuntia subulata ssp. exaltata } \\
\text { (Berger) D.Hunt }\end{array}$ & ancocasa \\
\hline Oroya peruviana (K.Sch.) B. \& R. & amancosao \\
\hline \multicolumn{2}{|l|}{ CAPRIFOLIACEAE } \\
\hline Sambucus peruviana Kunth. & rayán \\
\hline \multicolumn{2}{|l|}{ CARYOPHYLLACEAE } \\
\hline \multicolumn{2}{|l|}{ Drymaria sp. } \\
\hline \multicolumn{2}{|l|}{ Pycnophyllum glomeratum Mart. } \\
\hline \multicolumn{2}{|l|}{ CELASTRACEAE } \\
\hline Maytenus apurimacensis Loest. & tullma 2 \\
\hline \multicolumn{2}{|l|}{ CHENOPODIACEA } \\
\hline Chenopodium sp. & ccentilquinoa \\
\hline \multicolumn{2}{|l|}{ CONVOLVULACEAE } \\
\hline \multicolumn{2}{|l|}{ Ipomoea sp. } \\
\hline \multicolumn{2}{|l|}{ CRASSULACEAE } \\
\hline Villadia sp. & siempre viva \\
\hline \multicolumn{2}{|l|}{ CUCURBITACEAE } \\
\hline Cyclanthera brachybotrys (P. et End.) Cogn. & caigua silvestre \\
\hline \multicolumn{2}{|c|}{\begin{tabular}{l|l} 
ELAEOCARPACEAE & \\
\end{tabular}} \\
\hline Vallea stipularis L.f. & ccello ccoi \\
\hline \multicolumn{2}{|l|}{ EPHEDRACEAE } \\
\hline Ephedra americana H. \& B. ex Willd. & suelda con suelda \\
\hline EQUISETACEAE & \\
\hline
\end{tabular}




\begin{tabular}{|c|c|}
\hline Equisetum bogotense Kunth & condorpasarasara \\
\hline \multicolumn{2}{|l|}{ ERICACEAE } \\
\hline \multicolumn{2}{|l|}{ Pernettya prostrata (Cav.) D.C. } \\
\hline \multicolumn{2}{|l|}{ EUPHORBIACEAE } \\
\hline Euphorbia sp. & leche-leche \\
\hline \multicolumn{2}{|l|}{ FABACEAE } \\
\hline Adesmia sp. & tarwi silvestre \\
\hline Astragalus garbancillo Cav. & garbancillo \\
\hline Dalea peruviana (J.F. Macbr.) J.F. Macbr. & garbanzo silvestre \\
\hline Lupinus sp. & tarwi silvestre \\
\hline Medicago lupulina $\mathrm{L}$. & chinatrebol \\
\hline Otholobium pubescens (Poir.) Grim. & culén \\
\hline Senna sp. & mutuy enano \\
\hline Senna sp. & mutuy \\
\hline Trifolium sp. & tribol \\
\hline Vicia andicola H.B.K. & arverja silvestre \\
\hline \multicolumn{2}{|l|}{ GERANIACEAE } \\
\hline Erodium moschatum (L.) L'H.ex A. & aucca-aucca \\
\hline \multicolumn{2}{|l|}{ HYDROPHYLLACEAE } \\
\hline \multicolumn{2}{|l|}{ Phacelia secunda J.F.Gmel. } \\
\hline \multicolumn{2}{|l|}{ IRIDACEAE } \\
\hline \multicolumn{2}{|l|}{ Sisyrinchium chilense Hook. } \\
\hline \multicolumn{2}{|l|}{ JUNCACEAE } \\
\hline Distichia muscoides N \& M. & turbera \\
\hline Luzula aff.racemosa Desv. & chilhua \\
\hline \multicolumn{2}{|l|}{ LAMIACEAE } \\
\hline Lamium amplexicaule $\mathrm{L}$. & occeccora \\
\hline Marrubium vulgare $\mathrm{L}$. & ccentilpaitaña \\
\hline Mynthostachys mollis (Kunth.)Gris. & muña \\
\hline Salvia oppositiflora $\mathrm{R}$. et P. & soconpe \\
\hline Satureja boliviana Briq. & asnomuña \\
\hline Satureja incana (R. et P.) Spreg. & pishcopatacllan \\
\hline \multicolumn{2}{|l|}{ LILIACEAE } \\
\hline \multicolumn{2}{|l|}{ Bomarea aff.dulcis (Hook.) Beauv. } \\
\hline \multicolumn{2}{|l|}{ Bomarea ovata (Cav.) Mirb. } \\
\hline \multicolumn{2}{|l|}{ LINACEAE } \\
\hline Linum cf.oligophyllum Willd. ex.Sch. & piquipichana \\
\hline \multicolumn{2}{|l|}{ LOASACEAE } \\
\hline Caiophora cirsiifolia Pres1 & ortiga o pukasisa \\
\hline \multicolumn{2}{|l|}{ LOGANIACEAE } \\
\hline Buddleia coriacea. & ccolle \\
\hline Buddleia incana $\mathrm{R}$. et $\mathrm{P}$. & quisuar \\
\hline \multicolumn{2}{|l|}{ LORANTHACEAE } \\
\hline Psittacanthus cuneifolius (R.\&.P) G.Don & tullma \\
\hline \multicolumn{2}{|l|}{ MALVACEAE } \\
\hline Acaulimalva engleriana (Ulbr.) Krap. & realsaltia \\
\hline \multicolumn{2}{|l|}{ MYRTACEAE } \\
\hline Eucalyptus globulus Labill. & eucalipto \\
\hline ONAGRACEAE & \\
\hline
\end{tabular}

\begin{tabular}{|c|c|}
\hline Oenothera multicaulis R. et P. & yawarsocco \\
\hline Oenothera rosea L`H ex A. & chupasangre \\
\hline \multicolumn{2}{|l|}{ ORQUIDACEAE } \\
\hline$A a \mathrm{sp}$. & sara-sara \\
\hline Altensteinia sp. & boca de sapo \\
\hline \multicolumn{2}{|l|}{ OXALIDACEAE } \\
\hline Oxalis aff.dombei & oca silvestre \\
\hline Oxalis aff.nubigena Walp. & oca silvestre \\
\hline Oxalis aff.peduncularis Kunth & oca silvestre \\
\hline \multicolumn{2}{|l|}{ PASSIFLORACEAE } \\
\hline Passiflora tripartita var.mollisima Poir. & tumbo \\
\hline \multicolumn{2}{|l|}{ PIPERACEAE } \\
\hline Peperomia sp. & siempre viva \\
\hline \multicolumn{2}{|l|}{ PLANTAGINACEAE } \\
\hline \multicolumn{2}{|l|}{ Plantago aff.lanceolata } \\
\hline Plantago aff.australis Lam. & yanallantén \\
\hline Plantago linearis Knuth. & llantén \\
\hline Plantago rigida Kunth. & champa estrella \\
\hline \multicolumn{2}{|l|}{ Plantago sp. } \\
\hline Plantago sp1. & ancochuta \\
\hline \multicolumn{2}{|l|}{ POACEAE } \\
\hline Aciachne pulvinata Benth. & paco-paco \\
\hline \multicolumn{2}{|l|}{ Agrostis breviculmis Hitch. } \\
\hline Avena sp. & cebadilla \\
\hline Calamagrostis jamesonii Steud. & moqapasto \\
\hline Calamagrostis minima (Pilg.) Tovar & sora-sora \\
\hline \multicolumn{2}{|l|}{ Calamagrostis preslii (Knuth) Hitchc. } \\
\hline Calamagrostis rigescens (Presl) Scrib. & sora-sora \\
\hline \multicolumn{2}{|l|}{ Calamagrostis sp. } \\
\hline Calamagrostis spiciformis Hackel & soclla \\
\hline Calamagrostis vicunarum (Wedd.) Pilg. & crespillo \\
\hline Cortaderia jubata (Lem.) Stapf. & cola de zorro \\
\hline \multicolumn{2}{|l|}{ Festuca breviaristata Pilg. } \\
\hline Festuca dolichophylla Presl & chilhua \\
\hline Festuca horridula Pilg. & soclla \\
\hline Festuca weberbaueri Pilg. & chillhua \\
\hline \multicolumn{2}{|l|}{ Nasella meyeniana (Trin ex Rupr) Par. } \\
\hline \multicolumn{2}{|l|}{ Nasella pubiflora (T.et R.) Desv. } \\
\hline Nasella sp. & $\begin{array}{l}\text { paccpa cora o pasto } \\
\text { de la montaña }\end{array}$ \\
\hline Poa horridula Pilg. & $\mathrm{NN}$ \\
\hline \multicolumn{2}{|l|}{ Poa lilloi Hack. } \\
\hline \multicolumn{2}{|l|}{ Stipa ichu (R. et P.) Kunth. } \\
\hline Stipa mexicana Hitch. & soclla \\
\hline \multicolumn{2}{|l|}{ POLYGALACEAE } \\
\hline Monnina salicifolia $\mathrm{R}$. et $\mathrm{P}$. & totahuiña \\
\hline \multicolumn{2}{|l|}{ POLYGONACEAE } \\
\hline Muehlenbeckia volcanica (Bent) Eng. & muyaca \\
\hline Rumex sp. & rumanza \\
\hline POLYPODIACEAE & \\
\hline
\end{tabular}




\begin{tabular}{|c|c|}
\hline $\begin{array}{l}\text { Campyloneurum amphostenon (Kunze ex } \\
\text { Klotzsch) Fee. }\end{array}$ & jalahuala \\
\hline Campyloneurum densifolium (Hier.) Lell. & jala Huala \\
\hline Campyloneurum sp. & ccalahuala \\
\hline Polypodium buchtienii Rosenst. & raqui-raqui \\
\hline \multicolumn{2}{|l|}{ Polypodium sp. } \\
\hline \multicolumn{2}{|l|}{ PTERIDACEAE } \\
\hline Adiantum porreti W.Kstr. & culantrillo \\
\hline Argyrochosma nivea (Poir.) Wind. & helecho \\
\hline Cheilanthes incarum Maxon & coca de inca \\
\hline Cheilanthes pruinata Maxon & $\begin{array}{l}\text { culandrillo de pozo o } \\
\text { culantrillo macho }\end{array}$ \\
\hline \multicolumn{2}{|l|}{ RANUNCULACEAE } \\
\hline \multicolumn{2}{|l|}{ Clematis seemanni O.Ktze. } \\
\hline \multicolumn{2}{|l|}{ RHAMNACEAE } \\
\hline Colletia spinossisima J .F.Gmel. & occechica \\
\hline \multicolumn{2}{|l|}{ ROSACEAE } \\
\hline Hesperomeles aff.cuneata Lindl. & milo \\
\hline Kageneckia lanceolata R. et P. & lloque \\
\hline Polylepis racemosa R. et $\mathrm{P}$. & queñoal \\
\hline Prunus serotina var. salicifolia Ehrh. & capuli \\
\hline Tetraglochin cristatum(Brit.)Rothm. & rancha-rancha \\
\hline \multicolumn{2}{|l|}{ RUBIACEAE } \\
\hline Arcytophyllum thymifolium (R. et P.) Stand. & pichi-pichi \\
\hline Gallium corymbosum $\mathrm{R}$. et $\mathrm{P}$. & duraznito \\
\hline \multicolumn{2}{|l|}{ SANTALACEAE } \\
\hline Quichamallium sp. & chinchimalli \\
\hline \multicolumn{2}{|l|}{ SAXIFRAGACEAE } \\
\hline \multicolumn{2}{|l|}{ Ribes sp. } \\
\hline \multicolumn{2}{|l|}{ Saxifraga magellanica Poir. } \\
\hline \multicolumn{2}{|l|}{ SCROPHULARIACEAE } \\
\hline \multicolumn{2}{|l|}{ Agalinis lanceolata (R. et P.) D’Arcy } \\
\hline Alonsoa acutifolia $\mathrm{R}$. et P. & cuchu-cuchu \\
\hline \multicolumn{2}{|l|}{ Bartsia aff.weberbaueri Diels } \\
\hline \multicolumn{2}{|l|}{ Bartsia inaequalis Benth. } \\
\hline \multicolumn{2}{|l|}{ Bartsia melampyroides (H.B.K) Benth. } \\
\hline Calceolaria sp. & zapatito, huahuyllay \\
\hline Castilleja sp. & pimpinilla macho \\
\hline Veronica aff.persica. & occeccora \\
\hline \multicolumn{2}{|l|}{ SOLANACEAE } \\
\hline Dunalia aff.espinosa (Meyen) Dam. & ayantancar \\
\hline Solanum acaule Bitt. & atoccsiri \\
\hline \multicolumn{2}{|l|}{ Solanum aff.furcatum Dun. } \\
\hline Solanum bukasovii Juz. & papa del zorro \\
\hline Solanum bukasovii Juz. f. multidissectum. & atoccpapa \\
\hline \multicolumn{2}{|l|}{ Solanum glutinosum Dun. } \\
\hline Solanum multiinterruptum Bitt. & kìta mauna \\
\hline Solanum nitidum $\mathrm{R}$. et $\mathrm{P}$. & ñuñunga \\
\hline \multicolumn{2}{|l|}{ TROPAEOLACEAE } \\
\hline $\begin{array}{l}\text { Tropaeolum tuberosum subsp. silvestre } \\
\text { Sparre }\end{array}$ & k'ita añu \\
\hline
\end{tabular}

\begin{tabular}{|l|l|}
\hline VERBENACEAE & \\
\hline Citharexylum dentatum D.Don & lingli \\
\hline Duranta aff.armata Mold. & tancar \\
\hline Verbena litoralis Kunth. & verbina \\
\hline
\end{tabular}

\section{Formaciones Vegetales}

La presencia de ciertas formas de vida permitió diferenciar 8 formaciones vegetales con las siguientes especies dominantes (Figura 3, Tabla 3):

a. Oconal (4000- $4400 \mathrm{msnm})$

Posee plantas almohadilladas ubicadas en zonas con diferentes grados de humedad del suelo y pendientes entre 0 y $15^{\circ}$. Las especies características son Aciachne pulvinata, Pycnophyllum molle, Plantago sp. y Distichia muscoides. Para el caso de los dos tipos de oconales, predomina en densidad el Aciachne pulvinata. La extensión de oconales en la microcuenca es de gran importancia debido a formar parte de la fuente de origen del río Pachachaca, y por lo tanto, es la base del ciclo hidrológico de esta microcuenca. Así mismo, los oconales junto con los pastizales y pajonales constituyen áreas de pastoreo de alpacas y llamas.

b. Pastizal (4300 msnm)

Formación dominada por herbáceas de la familia Poaceae de porte muy reducido (menor a $2 \mathrm{~cm}$ de altura), acompañado de juncáceas pequeñas. Se halla en la parte alta de la microcuenca en zonas casi planas, cerca a los oconales. Pertenece a las áreas de pasturas. Aciachne pulvinata domina con una densidad mayor que sobre el resto de especies, como del género de Calamagrostis, entre otras.

c. Pajonal (3900 - $4200 \mathrm{msnm})$

Se encuentra en la parte alta de la microcuenca en zonas de pendiente entre 15 a $50^{\circ}$. Posee una gran densidad de individuos de Stipa ichu acompañado de arbustos de Astragalus garbancillo. Es una de las formaciones vegetales naturales que se encuentra más fragmentada a lo largo de la microcuenca debido al sobrepastoreo ocasionado mayormente por el ganado vacuno. Las especies dominantes son Stipa ichu, seguido de Festuca weberbaueri y A. garbancillo.

d. Matorral bajo sub-inerme (3850 - $3950 \mathrm{msnm})$

Formación vegetal constituida por matorrales bajos de menos de $1 \mathrm{~m}$, con pocas especies no palatables, principalmente Chuquiraga sp. pero con dominancia de Calceolaria sp. seguida de Astragalus garbancillo. Se encuentra en las zonas de transición de la parte media a la parte alta de la microcuenca, al final de un pequeño cañón que termina en la zona de pastos. Ubicada en zonas relativamente escarpadas, de pendiente entre 20 a $40^{\circ}$. En cuanto a la densidad, predomina Calceolaria sp. seguido de Chuquiraga sp. También pertenecen a esta formación una Lamiaceae como Minthostachys mollis y otras de la familia 
Asteraceae.

e. Matorral enano sub- inerme (3800 $\mathrm{msnm})$

Constituido por arbustos enanos de las especies Tetraglochin cristatum y Arcytophyllum thymifolium, principalmente sufrutescentes, inermes, es decir con una textura lisa, en la mayoría de ellos. Se le encuentra en la parte media de la microcuenca, en zonas de pendiente entre $15^{\circ}$ a $40^{\circ}$. Posiblemente fueron zonas de cultivos, terrenos en descanso o de rotación. Predomina el A. thymifolium sobre $T$. cristatum. En menor cantidad se pueden encontrar en este tipo de matorrales a especies como Astragalus garbancillo y Baccharis aff. caespitosa.

f. Matorral medio inerme (3700 $\mathrm{msnm}$ )

Formación vegetal constituida principalmente por arbustos medios de textura lisa. Representa la parte media de la microcuenca, con fuertes pendientes de 60 a $70^{\circ}$, donde la densidad más elevada la presenta Satureja incana, seguida de Aristeguieta discolor. En menores densidades se encuentran especies de Baccharis, Berberis y Monnina salicifolia.

g. Matorral medio espinoso (3450 - $3700 \mathrm{msnm})$

Constituido principalmente por arbustos espinosos o de hojas no palatables de porte mediano, donde domina el arbusto Duranta aff. armata. Esta formación se encuentra en dos lugares de la parte media de la microcuenca, en los cuales varia únicamente la presencia de algunas especies de baja densidad o cobertura. Se les halla sobretodo en laderas de pendiente entre 40 y $70^{\circ}$. Así también, se encontró en la parte baja de la microcuenca a 3450 msnm, dominada por la presencia de $D$. aff. armata aunque con especies diferentes a las dos zonas mencionadas anteriormente. En cuanto a densidad se observa la dominancia de $D$. aff. armata, seguido de especies como Gynoxys longifolia, Monnina salicifolia y Aristeguieta discolor. Otras especies que se pueden hallar en esta formación son Colletia spinossisima, Hesperomeles aff. cuneata, Citharexylum dentatum, Satureja incana, varias especies de Baccharis y una de Berberis, así como a Kageneckia lanceolata, Mutisia acuminata, Baccharis aff. salicifolia, Dunalia aff. spinosa y especies de Senna.

h. Matorral medio sub-inerme $(3470-3530 \mathrm{msnm})$ Está constituido por matorrales medios donde predominan aquellos espinosos, con una ligeramente menor presencia de arbustos inermes. Se encuentra en la parte baja de la microcuenca, en zonas de fuerte pendiente, de entre 40 a $50^{\circ}$. Se pueden encontrar especies como Satureja incana, Aristeguieta discolor, Kageneckia lanceolata, Colletia spinossissima, Hesperomeles aff. cuneata, Ephedra americana, Citharexylum dentatum, Berberis sp., Mintosthachys mollis, Baccharis sp. $y$ Gynoxys longifolia. En cuanto a las densidades, se observa la dominancia de $S$. incana, seguido de Monnina salicifolia y Duranta aff. armata.

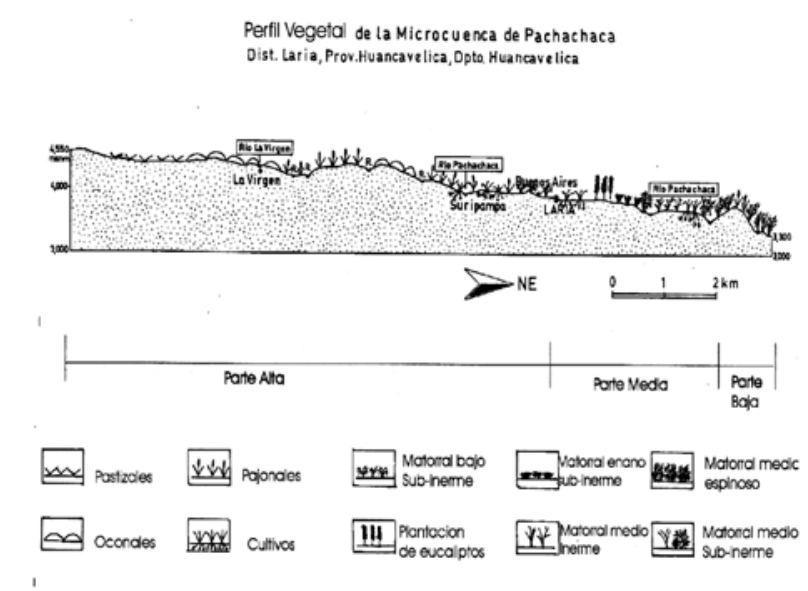

Figura 3. Perfil de la vegetación de la Microcuenca del río Pachachaca.

Tabla 2. Principales formas de vida de las especies vegetales de la Microcuenca del río Pachachaca. Huancavelica ( $\%$ de presencia).

\begin{tabular}{|c|c|c|c|}
\hline Formas de Vida & Parte Alta & Parte Media & Parte Baja \\
\hline Herbáceas & 30.8 & 35.0 & 45.3 \\
\hline Arbustivas & 18.2 & 65.9 & 59.1 \\
\hline Arbóreas & 28.6 & 57.1 & 28.6 \\
\hline Suculentas & 16.7 & 50.0 & 16.7 \\
\hline Epífitas & 0.0 & 40.0 & 100.0 \\
\hline
\end{tabular}

Tabla 3. Formaciones vegetales y especies dominantes de la Microcuenca del río Pachachaca. Huancavelica.

\begin{tabular}{|c|c|c|c|}
\hline $\begin{array}{l}\text { Formaciones } \\
\text { vegetales }\end{array}$ & $\begin{array}{l}\text { Altitud* } \\
\text { (m.s.n.m.) }\end{array}$ & Parte & Especies dominantes \\
\hline \multirow[b]{2}{*}{ Oconal } & 4300 & \multirow[b]{2}{*}{ Alta } & Aciachne pulvinata \\
\hline & 4400 & & $\begin{array}{l}\text { Aciachne pulvinata } \\
\text { Pycnophyllum molle }\end{array}$ \\
\hline Pastizal & 4300 & Alta & Aciachne pulvinata \\
\hline \multirow{2}{*}{ Pajonal } & 4150 & \multirow{2}{*}{ Alta } & $\begin{array}{l}\text { Stipa ichu } \\
\text { Astragalus garbancillo }\end{array}$ \\
\hline & 4050 & & $\begin{array}{l}\text { Festuca weberbaueri } \\
\text { Stipa ichu }\end{array}$ \\
\hline $\begin{array}{l}\text { Matorral bajo } \\
\text { sub-inerme }\end{array}$ & $\begin{array}{l}3870- \\
3900\end{array}$ & $\begin{array}{l}\text { Alta y } \\
\text { media }\end{array}$ & $\begin{array}{l}\text { Calceolaria sp. } \\
\text { Chuquiraga } \mathrm{sp} .\end{array}$ \\
\hline $\begin{array}{l}\text { Matorral enano } \\
\text { sub-inerme }\end{array}$ & 3800 & Media & $\begin{array}{l}\text { Arcythophyllum thymifolium } \\
\text { Tetraglochin cristatum }\end{array}$ \\
\hline $\begin{array}{l}\text { Matorral Medio } \\
\text { Inerme }\end{array}$ & 3700 & Media & $\begin{array}{l}\text { Satureja incana } \\
\text { Aristeguieta discolor }\end{array}$ \\
\hline \multirow{3}{*}{$\begin{array}{l}\text { Matorral medio } \\
\text { espinoso }\end{array}$} & 3450 & \multirow{3}{*}{$\begin{array}{l}\text { Media } \\
\text { y Baja }\end{array}$} & $\begin{array}{l}\text { Duranta } \text { aff.armata } \\
\text { Hesperomeles aff.cuneata }\end{array}$ \\
\hline & 3600 & & $\begin{array}{l}\text { Duranta } \text { aff.armata } \\
\text { Colletia spinossisima }\end{array}$ \\
\hline & 3700 & & $\begin{array}{l}\text { Duranta } \text { aff.armata } \\
\text { Berberis } \mathrm{sp} .\end{array}$ \\
\hline $\begin{array}{l}\text { Matorral medio } \\
\text { sub-inerme }\end{array}$ & $\begin{array}{l}3470- \\
3530\end{array}$ & Baja & $\begin{array}{l}\text { Satureja incana. } \\
\text { Duranta } \text { aff.armata }\end{array}$ \\
\hline
\end{tabular}

\section{CONCLUSIONES}

1. Se registraron a un total de 179 especies, correspon-dientes a 121 géneros y 57 familias.

2. Las familias mejor representadas fueron: Asteraceae, Poaceae y Fabaceae, con 30, 23 y 10 especies, respectivamente. 
3. La diversidad de especies disminuye según la altura, 87 en la parte baja y 45 en la parte alta de la microcuenca, presentándose un comportamiento similar con respecto a géneros y familias.

4. La forma de vida más representativa es la herbácea, la cual se encuentra extendida principalmente en la parte baja de la microcuenca, seguida por la arbustiva, cuya mayor cantidad de representantes se encuentra en la parte media.

5. Las especies arbóreas nativas han sido desplazadas por especies como Eucalyptus globulus y Prunus serotina. Se determinaron únicamente 3 especies nativas: Polylepis racemosa, Buddleia coriacea y Buddleia incana.

6. Se identificaron 8 formaciones vegetales naturales: oconal, pastizal, pajonal, matorral bajo sub-inerme, matorral enano sub-inerme, matorral medio inerme, matorral medio espinoso y matorral medio sub-inerme.

7. Los posibles parientes silvestres de cultivos nativos determinados fueron: Solanum bukasovii, Solanum acaule, Solanum multiinterruptum, Oxalis aff. peduncularis, Oxalis aff. nubigena, Ullucus sp., Tropaeolum tuberosum spp. silvestre y Chenopodium sp.

\section{Agradecimientos}

Los autores expresan su agradecimiento al Equipo de la Coordinadora de Ciencia y Tecnología en los Andes (CCTA); a los amigos de Talpuy-Huancayo; al Dr. Oscar Tovar, a la Dra. Blanca León y Dra. Graciela Vilcapoma por su apoyo; al Herbario USM de la Universidad nacional Mayor de San Marcos, al Herbario MOL de la Universidad Nacional Agraria La Molina; al Grupo de Ecología de Montañas 2001-II; compañeros de la UNALM que apoyaron en la fase de campo y a los amigos de las comunidades de Laria, Zunipampa, Mariscal Cáceres y Río de la Virgen, colaboradores en este trabajo.

\section{Literatura citada}

Barrera J. \& Rubina A. 2000. Atlas de Huancavelica. Desco. Perú.

Brako L. \& Zarucchi J. 1993. Catalogue of the Flowering Plants and Gymnosperms of Perú. Monograhs in Systematic Botany from the Missouri Botanical Garden. Vol 45.

Martínez S., Tovar O. \& Galán A. 1989. Pisos bioclimáticos y cultivos del Perú. Informe final de Proyecto. CEDINFOR. Lima. Perú.

Ochoa C. 1999. Las papas de Sudamérica. CIP
Ediciones. Lima, Perú.

OFICINA NACIONAL DE EVALUACIÓN DE RECURSOS NATURALES (ONERN). 1976. Mapa Ecológico del Peru. Guía Explicativa. ONERN. Lima, Perú.

OFICINA NACIONAL DE EVALUACIÓN DE RECURSOS NATURALES (ONERN). 1984. Inventario, evaluación de recursos naturales de la zona altoandina del Perú (Departamento de Huancavelica). Lima- Perú

Parra F. 2003. Composición floristica y estructura de la vegetación de la Microcuenca del Pachachaca, Distrito de Laria, Provincia de Huancavelica, Departamento de Huancavelica. Tesis para optar por el título de Bióloga. UNALM.

TALPUY-UNALM-LARIA-ZUNIPAMPA-BUENOS AIRES-RIO DE LA VIRGEN. 2001. Pachachaca Huaycco. Informe Final del Curso de Ecología de Montañas.

Torres J. 1987. Ecología y Uso de los Recursos Naturales renovables de la Cuenca del Rio San Marcos, Tamaulipas, México. Tesis para obtener el grado de Maestro en Ciencias (Biología). Facultad de Ciencias. Universidad Autónoma de México. México

Tosi J.A. 1960. Zonas de Vida Natural en el Perú. Memoria explicativa sobre el Mapa Ecológico del Perú. Instituto Interamericano de Ciencias Agrícolas de la Zona Andina. Proyecto 29. Programa de Cooperación Técnica. Boletín Técnico $\mathrm{N}^{\circ} 5$.

Tovar O. 1957. Las gramíneas de Huancavelica. Memorias del Museo de Historia Natural "Javier Prado". Lima. Perú.

1990. Tipos de vegetación, diversidad florística y estado de conservación de la Cuenca del Mantaro. Centro de Documentación para la Conservación CDC, UNALM. Lima Perú.

1993. Las gramíneas (Poaceae) del Perú. Ruizia. Monografías del Real Jardín Botánico y CSIC. Tomo 13. Madrid, España.

Vila M. 1997. Estructura y Análisis de la vegetación de la microcuenca de Huarmiragra, Distrito de Tomayquichua, Provincia de Ambo, Departamento de Huánuco. Tesis para optar por el título de Bióloga. UNALM.

Weberbauer A. 1945. El Mundo Vegetal de los Andes Peruanos. Estudio Fitogeográfico. 2da Edición. Estación Experimental Agrícola de La Molina. Dirección de Agricultura. Ministerio de Agricultura. Lima.

\footnotetext{
${ }^{1}$ Departamento Académico de Biología. Universidad Nacional Agraria La Molina. Av. La Universidad s/n. quisuar@yahoo.com / amotape@yahoo.com / aceroni@lamolina.edu.pe. Apartado postal 12-056. Lima 12-Perú. Fax: 3496015.
} 